\title{
Caracterización de la conducta adaptativa en escolares con y sin discapacidad intelectual de la región de la Araucanía*
}

\author{
Characterization of adaptive behavior \\ in students with and without intellectual disabilities \\ in the araucanía region
}

\begin{abstract}
Arlett Krause Arriagada**
Francisca Román Mella

Yennifer Esparza Parra

Marjorie Novoa Mercado

Pamela Salinas Pulleghini

Fernanda Toledo Padilla

Arlyn Vallejos Medina Universidad de La Frontera,

Temuco, Chile

Recibido: 20 de junio de 2015 Revisado: 30 de agosto de 2015 Aceptado: 13 de octubre de 2015
\end{abstract}

\section{Resumen}

Esta investigación se enfoca en la caracterización de la conducta adaptativa en escolares con y sin discapacidad intelectual de la Región de La Araucanía, Chile. Se utilizó el Inventario para la Planificación de Servicios y Programación Individual (ICAP), aplicado a padres y/o tutores legales de 22 niños con discapacidad intelectual y 33 niños sin discapacidad. Las edades fluctuaron entre los 6 y 12 años, con un diseño no probabilístico en conglomerados. El método utilizado es descriptivo-comparativo. Los resultados señalan que existen diferencias de las conductas adaptativas, donde los escolares sin discapacidad presentan un mayor nivel de desarrollo, además ambos grupos poseen un perfil heterogéneo en las destrezas que componen el funcionamiento adaptativo, donde las destrezas motoras son las más altas, mientras que las destrezas de la vida en comunidad son las más bajas. Se concluye que las diferencias encontradas se ven afectadas por la presencia de

Artículo de investigación. Producto del proyecto de investigación Diufro DI12-0077 (2012-2013). Dirección de Investigación Universidad de La Frontera, Temuco, Chile. Nombre proyecto: "Propiedades psicométricas de la versión española del Inventario para la Planificación de Servicios y la Programación Individual (ICAP), en escolares de la ciudad de Temuco". http://dx.doi.org/10.15332/s1794-9998.2016.0001.02 Correspondencia: Arlett Krause Arriagada, Departamento de Psicología, Universidad de La Frontera. Dirección postal: avenida Francisco Salazar 01145, casilla 54-D, Temuco, Chile. Correo electrónico: arlett.krause@ufrontera.cl 
discapacidad intelectual y por el contexto educativo. Esta investigación se enmarca en el proyecto de investigación DI12-0077 denominado Propiedades psicométricas de la versión española del Inventario para la planificación de servicios y la programación individual (ICAP), en escolares de la ciudad de Temuco.

Palabras clave: conducta adaptativa, discapacidad intelectual, escolares.

\section{Abstract}

This investigation focused on the characterization of adaptive behavior in students with or without intellectual disabilities in the Araucanía Region in Chile. The Inventory for Service Planning and Individual Programming (ICAP) was used with a sample of parents or legal tutors of 22 children with intellectual disabilities and 33 children without disabilities. The ages of the students ranged between 6 and 12 years, with a non-probabilistic cluster sampling design, with descriptive-comparative methods for analysis. Results show differences in adaptive behavior between the assessed groups, where students without disabilities show a higher level of development. Also both groups exhibit heterogeneous skill profiles, with motor skills being the most developed, and life skills in community being the least developed. These differences might have been affected by the presence of intellectual disabilities and by the educational contexts. This study is associated to the DI120077 research project entitled "Psychometric Properties of the Spanish version of the inventory for service planning and individual programming (ICAP), in students of the city of Temuco".

Keywords: Adaptive behavior, intellectual disability, scholar.

\section{Introducción}

En la sociedad chilena el grupo de personas con discapacidad corresponde al $12,93 \%$ de la población, cifra que aumenta en los sectores de menores recursos, situando a estos individuos en los grupos sociales más vulnerables del país (Lotito y Sanhueza, 2011). Es por esta situación que en el año 2000 se realiza el Primer estudio nacional de la discapacidad en Chile, realizado por el Instituto Nacional de Estadísticas (INE, 2004), el cual indica que el $34,6 \%$ de los hogares de nuestro país tiene entre sus miembros al menos una persona que presente discapacidad. En este sentido, se torna necesario que en el país se implementen medidas de inclusión para este grupo, ya que hasta entonces la mayoría de los ámbitos del funcionamiento social y comunitario en Chile, han sido diseñados para funcionar en áreas de educación, cultura, transporte, entre otros, sin considerar a este conjunto de personas.
Actualmente, a través de la reforma educacional, se han desarrollado políticas y lineamientos para las personas con discapacidad, orientados a mejorar su calidad de vida, equiparando las oportunidades educativas a las que puedan acceder. El programa de educación especial del Ministerio de Educación (MINEDUC), es el responsable hasta ahora de implementar políticas de atención en todos los niveles y modalidades del sistema escolar (Godoy, Meza y Salazar, 2004), para personas con Necesidades Educativas Especiales (NEE), que se definen como dificultades que afectan a todas 0 algunas áreas del aprendizaje del alumno y que pueden ser de carácter transitorio o permanente (Ayala, 2012). Ante esta situación, se torna necesario contar con instrumentos que permitan la evaluación del funcionamiento adaptativo de personas con o sin discapacidad y así optimizar los planes de acción para mejorar su situación educativa. En este sentido, el MINEDUC recomienda el uso de la Escala de Madurez Social de Vineland, 
que data del año 1959, sin embargo esta adaptación realizada por Otero Quiroz, no presenta baremos para la población nacional (MINEDUC, 2009). Es por esto que surge la necesidad de adaptar instrumentos para evaluar el funcionamiento adaptativo en nuestra población.

En este contexto, un grupo de profesionales de la Universidad de La Frontera está desarrollando el proyecto de investigación "Propiedades psicométricas de la versión española del Inventario para la Planificación de Servicios y la Programación Individual (ICAP), en escolares de la ciudad de Temuco", con el fin de determinar las propiedades psicométricas de este instrumento, que mide funcionamiento adaptativo y problemas de conducta. Es así como surge la inquietud de profundizar sobre esta temática y por ende el presente estudio se propuso como objetivo caracterizar la conducta adaptativa en escolares con y sin discapacidad intelectual en la Región de La Araucanía, donde el análisis de los datos recogidos permite contar con mayores antecedentes a la hora de tomar decisiones para la planificación de ayudas o apoyo pedagógico para niños con NEE integrados en colegios regulares, como también en escuelas especiales. Además, se pretende contribuir a la generación de nuevos conocimientos, puesto que no se cuenta con estudios chilenos publicados que caractericen la conducta adaptativa actualizados al contexto nacional.

En la historia de la humanidad siempre han existido personas con discapacidad intelectual, de modo que la sociedad se constituye por miembros con diversas capacidades que van evolucionando según las necesidades, las expectativas y la consciencia social (Medina, 2010). De este modo, el presente estudio contempló como conceptos esenciales la discapacidad, discapacidad intelectual y conducta adaptativa. Según Schalock et al. (2007):

El constructo actual de discapacidad se centra en la expresión de limitaciones en el funcionamiento individual en un contexto social y que representa una desventaja substancial para el individuo. Tiene su origen en una condición de salud que da lugar a déficit en el cuerpo y las estructuras, limitaciones en la actividad y restricciones en la participación en el contexto de 1os factores personales y ambientales (p. 6).

Al respecto, Luckasson et al. (2002) puntualizan la discapacidad intelectual como:

La presencia de limitaciones sustantivas en el funcionamiento actual del estudiante, caracterizado por un desempeño intelectual significativamente por debajo de la media que se da en forma concurrente con limitaciones en su conducta adaptativa, manifestada en habilidades prácticas, sociales y conceptuales, y que comienza antes de los 18 años (p. 8).

Esta definición parte de cinco premisas esenciales para su aplicación, según MINEDUC (2009):

1. Las limitaciones en el funcionamiento presente deben considerarse en el contexto de ambientes comunitarios típicos de los iguales en edad y cultura.

2. Una evaluación válida ha de tener en cuenta la diversidad cultural y lingüística, así como las diferencia en comunicación y en aspectos sensoriales, motores y comportamentales.

3. En un individuo las limitaciones a menudo coexisten con capacidades.

4. Un propósito importante de describir limitaciones es el desarrollar un perfil de los apoyos necesarios.

5. Si se ofrecen los apoyos personalizados apropiados durante un periodo prolongado, el funcionamiento en la vida de la persona con retraso mental generalmente mejorará.

El concepto de conducta adaptativa hace alusión "al conjunto de habilidades conceptuales, sociales y prácticas aprendidas por las personas para funcionar en su vida diaria" (Luckasson et al., 2002, p. 73). Las habilidades conceptuales engloban el lenguaje (expresivo y receptivo), lectura y escritura, conceptos de dinero, autodirección, es decir factores cognitivos, de comunicación y habilidades académicas; las habilidades sociales implican responsabilidad, autoestima, habilidades 
interpersonales, credulidad (probabilidades de ser engañado o manipulado) e ingenuidad, que son necesarias para las relaciones sociales y las competencia emocionales; por último, las habilidades prácticas conllevan las actividades de la vida diaria (comida, movilidad, aseo, vestido), actividades instrumentales (preparación de comidas, mantenimiento de la casa, transporte, toma de medicinas, manejo del dinero), habilidades ocupacionales y mantención de entornos seguros, las cuales están dadas por la capacidad física para el mantenimiento de la persona en actividades profesionales de la vida diaria (Navas, Verdugo, Arias y Gómez, 2010).

García (2001) señala que la conducta y las habilidades adaptativas van progresando conforme lo hace la edad cronológica de los sujetos con y sin retraso mental. En particular, los sujetos con retraso mental presentan una discontinuidad en la adquisición de estas habilidades, por lo que se deduce poseen un ritmo más irregular que el de las personas sin retraso. Por otro lado, se ha encontrado mayor dispersión en los datos obtenidos de personas con retraso mental, lo que demuestra una gran variabilidad en las conductas y áreas adaptativas.

Hernández y Ramírez (2007), evidencian que el $56 \%$ de los niños se encontraban medianamente adaptados, un $27 \%$ se encontraban escasamente adaptados y el $17 \%$ restante estaban suficientemente adaptados. Además, se muestra que las características familiares no ejercen una influencia significativa en el nivel de conducta adaptativa que los niños desarrollen, sino que estaría directamente relacionada con el grado de deficiencia mental que estos presentan. Finalmente se observó que las habilidades prácticas son las que presentan un mayor porcentaje de adaptación.

Medina (2010), concluye que a mayor capacidad intelectual, hay un mayor uso de la conducta adaptativa y también determina que las medias más bajas se encuentran en el dominio de independencia $(92,73)$, destrezas económicas $(9,99)$, desarrollo del lenguaje $(28,36)$ y actividades domésticas $(10,21)$, que corresponden a las áreas donde los adultos presentan mayores limitaciones.
Además, se señala que la conducta adaptativa disminuye según aumenta la edad de las personas afectadas, es así como el rango de edad de los 20 a 30 años obtiene una media de 253,95, que decae al llegar al rango de los 61 a 80 años con una media de 192,78.

En España, García, de la Fuente y Fernández (2010), encontraron que los niños con discapacidad intelectual tienen un proceso más lento en la adquisición y el uso del comportamiento adaptativo en relación a los niños sin discapacidad $(x=105)$. Además, los niños con discapacidad intelectual leve obtienen resultados más altos que los niños con una discapacidad intelectual moderada $(x=89$ y $x=81)$, y estos últimos, a su vez, consiguen puntuaciones más altas que los niños con una discapacidad intelectual grave $(x=42)$. De acuerdo a lo anterior, se propuso la siguiente pregunta de investigación: ¿existen diferencias en las conductas adaptativas de los escolares con y sin discapacidad de la región de La Araucanía?

Se planteó como objetivo general caracterizar la conducta adaptativa en escolares con y sin discapacidad en la región de La Araucanía.

Como objetivos específicos se pretendió:

1. Analizar si existen diferencias entre escolares con y sin discapacidad intelectual respecto de su conducta adaptativa.

H1: "existen diferencias en la conducta adaptativa de escolares con y sin discapacidad".

2. Determinar el perfil de desarrollo de las áreas de la conducta adaptativa en escolares con discapacidad.

H2: "los escolares con discapacidad presentan un desarrollo heterogéneo en las áreas de la conducta adaptativa".

3. Determinar el perfil de desarrollo de las áreas de la conducta adaptativa en escolares sin discapacidad.

H3: "los escolares sin discapacidad presentan un desarrollo homogéneo en las áreas de la conducta adaptativa". 


\section{Método}

\section{Participantes}

Se utilizó un muestreo no probabilístico por cuota, puesto que los miembros de la muestra no tuvieron la misma probabilidad de ser seleccionados. Fueron agrupados en estratos por las características deseadas de la investigación, pero estos no se seleccionaron aleatoriamente (Salkind, 1998). La muestra estuvo conformada en total por 55 personas, 33 padres o tutores legales de niños sin discapacidad, y 22 de niños con discapacidad intelectual, pertenecientes al primer ciclo escolar, los cuales se encontraron en escuelas normativas, especiales y programas de integración de la región de La Araucanía.

\section{Diseño}

El estudio fue comprendido desde una perspectiva cuantitativa y de acuerdo al objeto de investigación corresponde a un diseño descriptivo-comparativo. Descriptivo, pues se buscó especificar las propiedades importantes de un grupo de personas (Dankhe, 1989) de forma objetiva y comprobable (Colás, 1993), a través de la utilización del ICAP. Asimismo, se compararon las conductas adaptativas entre los niños con y sin discapacidad intelectual, a través de la información entregada por los padres o tutores legales.

\section{Instrumento}

Para la evaluación de la conducta adaptativa se utilizó el Inventario para Planificación de Servicio y Programación Individual (ICAP), versión española de Montero (1996), adaptado idiomáticamente por las investigadoras responsables del proyecto de investigación (Krause y Román, 2012). Este es un instrumento estructurado, que valora las distintas áreas del funcionamiento adaptativo y las necesidades de servicio de una persona. Se compone de un registro sistemático de datos relevantes (datos personales y limitaciones funcionales) y un test de conducta adaptativa que mide el nivel de las personas en relación a destrezas básicas para desenvolverse con independencia en su entorno. Se estructura en cuatro escalas: a) destrezas sociales y comunicativas (lenguaje expresivo y receptivo), b) destrezas de la vida personal (satisfacción de manera independiente de las necesidades personales más inmediatas), c) destrezas de vida en la comunidad (desenvolvimiento en actividades prácticas) y d) destrezas motoras (motricidad fina y gruesa). Es una prueba relativamente sencilla, rápida y de autoadministración a los padres o tutores legales de niños con y sin discapacidad. En el proceso de adaptación en España, presentó adecuados niveles de fiabilidad (Alpha de Cronbach), los cuales oscilaron entre el 0,86 a 0,98 en el caso de personas sin discapacidades y entre el 0,88 y el 0,98 en el caso de personas con discapacidades.

\section{Resguardos éticos}

Se tomaron los resguardos éticos de acuerdo al código de ética profesional del Colegio de Psicólogos de Chile, así se contó con un consentimiento informado, en el cual se explicitó el propósito general de la investigación, la relevancia y voluntariedad de la participación, además de la garantía de confidencialidad.

\section{Procedimiento}

Se contactó a los directores de cada establecimiento para coordinar una reunión donde se aplicó el instrumento. Primero, se les entregó el consentimiento informado a cada apoderado y luego se dieron las instrucciones para responder el ICAP. Cada aplicación tuvo un tiempo de duración aproximado de 40 minutos.

\section{Análisis de datos}

Se utilizó el paquete estadístico SPSS 20.0, con el cual se realizó un análisis estadístico de nivel descriptivo, a través de las medidas de tendencia central. Además, se estudió la distribución de las puntuaciones en la escala de la muestra por medio del Test de Shapiro-Wills que indicó el uso de pruebas paramétricas. Se utilizaron la prueba $t$ para muestras independientes y ANOVA de un factor para comprobar las hipótesis de investigación (Visauta, 2007). 


\section{Resultados}

La presentación de los resultados se realiza en tres fases: la primera corresponde a la confiabilidad del instrumento, la segunda a la descripción de los datos sociodemográficos y la tercera fase muestra la verificación de las hipótesis, a través del programa SPSS, versión 19.0.

\section{Fase 1}

El Inventario para Planificación de Servicio y Programación presenta adecuados niveles de fiabilidad (Alpha de Cronbach), los que oscilan entre 0,94 y 0,95 en relación a las dimensiones que mide la prueba.

\section{Fase 2}

Con respecto a la descripción de los datos sociodemográficos, la muestra estuvo constituida por un total de 55 padres o tutores legales de niños con y sin discapacidad intelectual, quienes entregaron información de sus hijos a través del ICAP. En relación al tipo de muestra, esta se conformó por 33 niños de población regular y 22 de población clínica. El sexo de la muestra se compuso en un $50,9 \%$ (28) por el sexo masculino y el 49,1 \% (27) restante pertenece al sexo femenino. La edad promedio de los niños fue de 8 años $(D E=1,575)$, con un rango que va desde los 6 a 12 años.

En relación al nivel de discapacidad mental de la muestra, se observó que el 60 \% (33) de la población no presentó discapacidad intelectual, mientras que el resto de la muestra sí presentó niveles de discapacidad intelectual, los que se dividen en leve $16,4 \%$ (9), moderada $9,1 \%$ (5), severo 3,6 \% (2), profundo $1,8 \%$ (1) y desarrollo lento o de riesgo con $7,3 \%$ (4). Cabe destacar, que los niveles de discapacidad intelectual fueron reportados por los padres o tutores legales, de acuerdo a la información de evaluaciones previas a la presente investigación.

\section{Fase 3}

En relación a las diferencias de la conducta adaptativa entre escolares con y sin discapacidad intelectual, se segmentó la población según el tipo de muestra y se aplicó la prueba t para muestras independientes, con la variable de independencia general. La muestra clínica presentó una media de $1,27(D E=0,594)$ y la muestra regular 2,39$\}$ $(D E=0,289)$, lo que indicó que existen diferencias significativas en la conducta adaptativa entre los niños con y sin discapacidad intelectual (ver figura 1$)$, con un $t(27,677)=-8,225 ; p=0,000) 95 \%$ IC [-1,401- -,842], por lo cual se aceptó la hipótesis de investigación.

Con respecto al desarrollo de las destrezas de la conducta adaptativa en escolares con discapacidad, se aplicó ANOVA de un factor que muestra diferencias significativas inter-grupo $(F=12,259$; $p=0,000$ ), para observar estas diferencias se aplicó la prueba post hoc de Tukey, que indicó que las áreas más desarrolladas son las destrezas motoras $(\bar{X}=1,70)$, las destrezas sociales y comunicativas $(\bar{X}=1,51)$ y las destrezas en la vida personal $(\bar{X}=$ $1,28)$, a excepción de las destrezas en la vida en comunidad donde la media es de 0,59 , siendo esta la menos desarrollada por la muestra clínica (ver figura 2). Lo anterior permite aceptar la hipótesis de investigación que nos indica un desarrollo heterogéneo de las destrezas de la conducta adaptativa en esta muestra.

En relación al desarrollo de las destrezas de la conducta adaptativa en escolares sin discapacidad, se aplicó ANOVA de un factor, que indicó diferencias significativas inter-grupo $(F=53,055$; $p=0,000$ ). Para observarlas, se aplicó una prueba post hoc de Tukey que indicó que las áreas más desarrolladas son las destrezas motoras $(\bar{X}=2,81)$ y las destrezas sociales y comunicativas $(\bar{X}=2,63)$, mientras que las destrezas en la vida personal presentaron una media de 2,36. Sin embargo, las destrezas en la vida en comunidad mostraron una media de 1,76 , siendo esta área la menos desarrollada por la muestra regular (ver figura 3). 
Figura 1.

Media del índice de independencia general, según tipo de muestra.

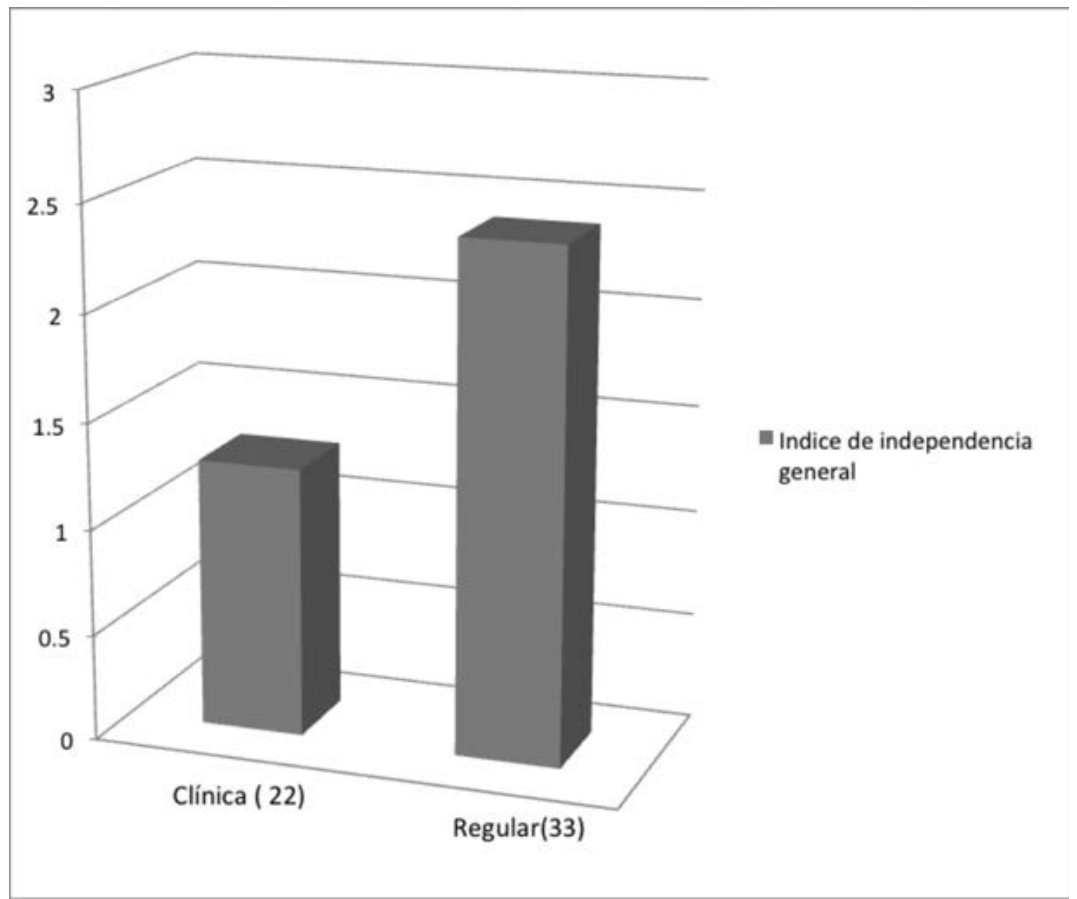

Figura 2.

Perfil de desarrollo de las destrezas de la conducta adaptativa, según sus medias en muestra clínica.

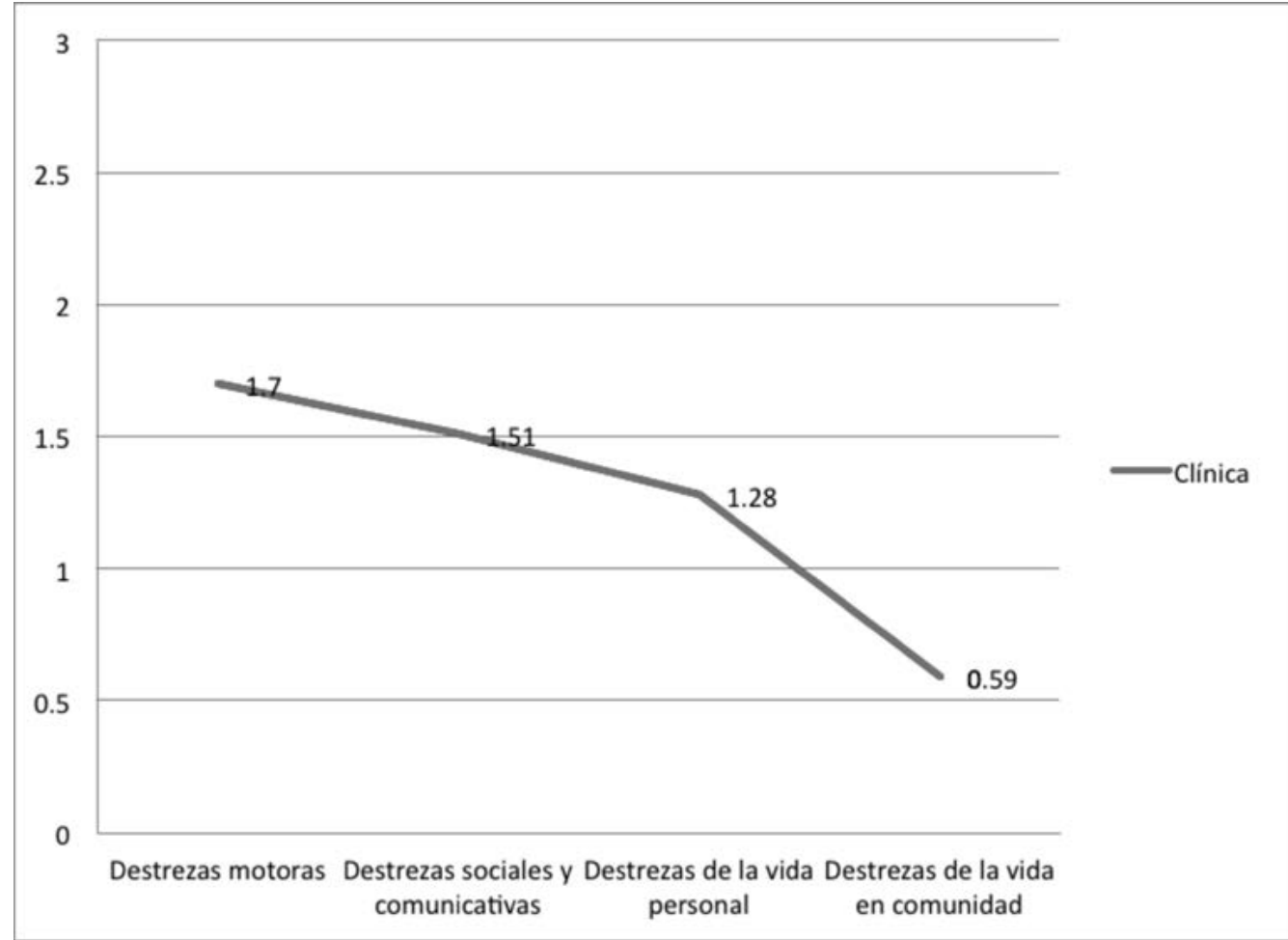


Figura 3.

Perfil de desarrollo de las destrezas de la conducta adaptativa, según sus medias en muestra regular.

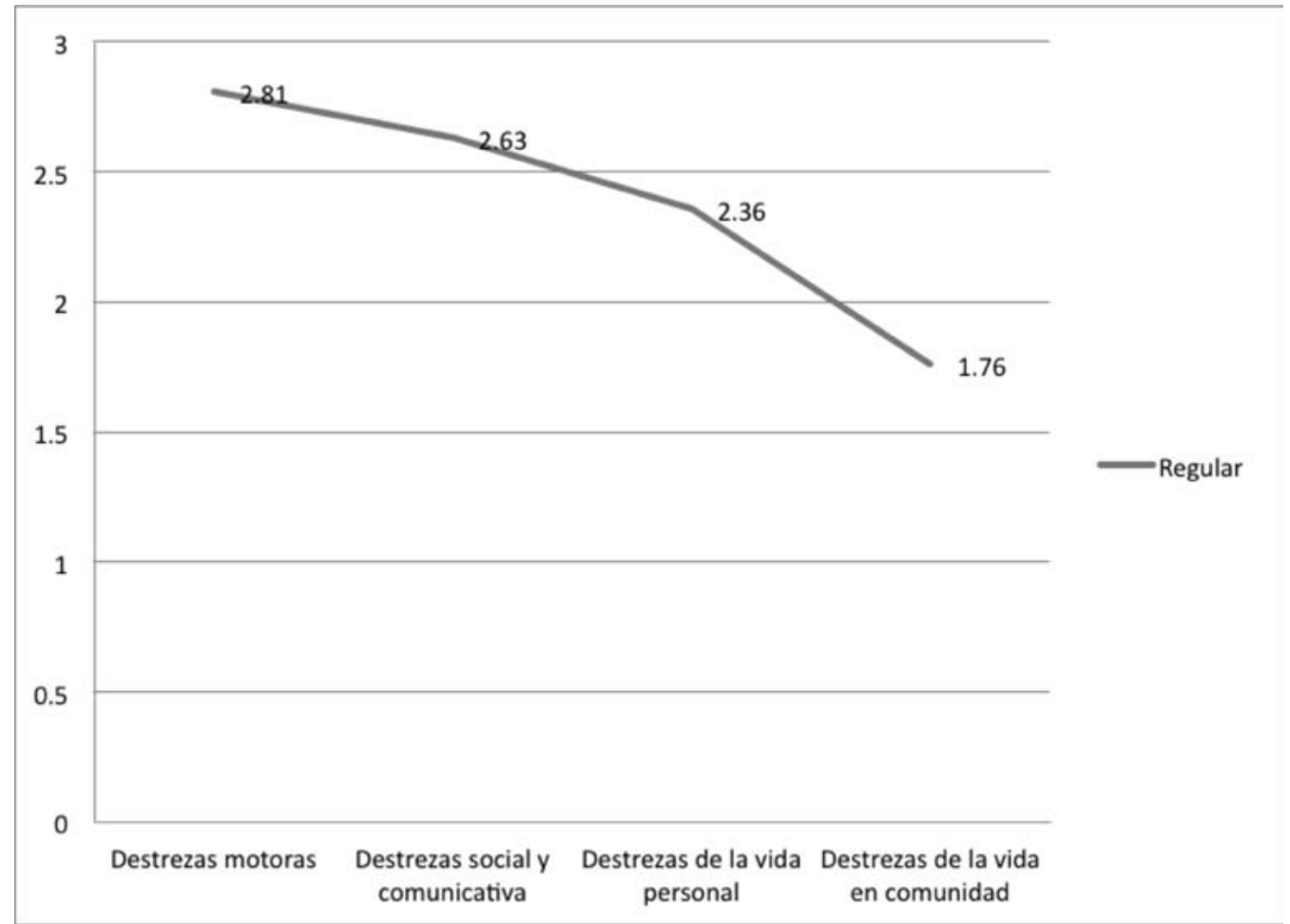

Lo anterior, muestra que existen diferencias significativas en el desarrollo de las destrezas que componen la conducta adaptativa, por lo cual se asumió la hipótesis nula, la cual indica que la conducta adaptativa presenta un desarrollo heterogéneo en los escolares sin discapacidad.

\section{Discusión}

El objetivo general de la presente investigación consistió en caracterizar la conducta adaptativa en escolares con y sin discapacidad de la región de La Araucanía. Este estudio ha permitido confirmar que existen diferencias significativas entre ambas poblaciones en relación a la conducta adaptativa, evidenciándose un mayor nivel de desarrollo de ésta en los escolares sin discapacidad. Estos hallazgos coinciden con los resultados obtenidos por García, de la Fuente y Fernández (2010), en relación a que los niños con discapacidad intelectual presentan un proceso más lento en la adquisición y el uso del comportamiento adaptativo en relación a sus pares convencionales, donde además el desarrollo de este comportamiento se encontraría ligado al nivel de discapacidad intelectual que el niño presente (Hernández y Ramírez, 2007).

Además, los niños con discapacidad presentan un desarrollo heterogéneo en las destrezas que componen el comportamiento adaptativo, es decir, las áreas evaluadas no presentan un desarrollo armónico. Lo cual concuerda con la evidencia empírica que hace mención de una mayor dispersión y gran variabilidad en las áreas que componen la conducta adaptativa (García, 2001). Lo anterior reafirma que la discapacidad intelectual en un factor influyente en la adquisición y uso del funcionamiento adaptativo.

En relación a los niños sin discapacidad intelectual que participaron de la investigación, se demuestra que poseen un desarrollo heterogéneo en el funcionamiento adaptativo al igual que sus 
pares con discapacidad intelectual, donde uno de los hallazgos más importantes es que ambas poblaciones presentan una tendencia similar en el perfil de desarrollo de las áreas que componen la conducta adaptativa. Este hecho se contrapone al estudio realizado por García (2001), ya que la investigación indica que las personas sin discapacidad poseen un ritmo más regular en la adquisición de la conducta y habilidades adaptativas en relación a los niños con discapacidad intelectual. Esta similitud en el desarrollo de las destrezas entre ambos grupos de la investigación, invita a reflexionar acerca de otras dimensiones que pueden influir en la adquisición y desarrollo de la conducta adaptativa del niño, como por ejemplo, las variables contextuales y familiares.

Se concluye que los escolares con y sin discapacidad poseen un desarrollo del funcionamiento adaptativo similar, que en ambos casos podría verse afectado por el contexto educativo que propicia el desarrollo de habilidades lógico matemáticas, más que enfocarse al desarrollo de competencias que permitan desenvolverse en la comunidad, lo que podría explicar que estas destrezas se encuentran menos desarrolladas independiente del desarrollo intelectual que el niño posea.

A pesar de abarcar una muestra acotada, que podría ser considerada una limitación, los resultados obtenidos concuerdan con la evidencia empírica de estudios similares, sin embargo, en el caso de los escolares sin discapacidad se encontró nueva información referente a que el desarrollo de los dominios de la conducta adaptativa en estos niños no seguiría una tendencia armónica.

Además, al trabajar con las apreciaciones que poseen los padres respecto al funcionamiento adaptativo de sus hijos, estas pueden incidir en una sobrevaloración o subestimación de su conducta adaptativa, situación dada por las características del instrumento utilizado, ya que no se complementa la información entregada por el padre o tutor legal del niño, por lo que es necesario que para futuras investigaciones se incluya la percepción de otras personas que interactúen regularmente con el evaluado, tales como, profesores, otros familiares, hermanos, entre otros; que permitan enriquecer la evaluación del funcionamiento adaptativo del escolar.

Otra posible línea de investigación apuntaría a indagar si estas diferencias contextuales son explicables con base a la dependencia de los colegios regulares (particulares, subvencionados o municipales) y a los tipos de escuela que asisten los estudiantes con discapacidad (escuela con programa de integración o escuelas especiales).

Finalmente, sería importante que las escuelas regulares fortalecieran el currículum de competencias adaptativas en los escolares de educación básica, enfocándose en el desarrollo y promoción de habilidades sociales que les permitan desenvolverse con autonomía dentro de la comunidad.

\section{Referencias}

Ayala, B. (2012). La motricidad en alumnos con necesidades educativas especiales. Análisis de seis casos. (Tesis de magíster). Recuperado de http://reunir.unir.net/handle/123456789/1289

Colás, B. M. (1993). Diseños de investigación para su aplicación a la evaluación de programas. Una guía práctica. Sevilla: Editorial Kronos.

Dankhe, G. (1989). Investigación y comunicación. La comunicación humana: ciencia social. México, D. F.: McGraw-Hill de México, pp. 385454.

García, M. I. (2001). Las personas con retraso mental y su diagnóstico: Traducción, adaptación y valoración de la "escala de conducta adaptativa ABS-S2" y del método de evaluación de las áreas de habilidades adaptativas. (Tesis de doctorado). España: Universidad de Burgos.

García, I., de la Fuente, R. \& Fernández, M. (2010). Adaptation of the ABS-S: 2 for use in Spain with children with intellectual disabilities. Policy and Practice in Intellectual Disabilities, 7(3), 221-230. 
Godoy, M., Meza, M. y Salazar, A. (2004). Antecedentes históricos, presente y futuro de la educación especial en Chile. Santiago, Chile: Ministerio de educación.

Hernández, A. y Ramírez L. (2007). Funcionamiento familiar y conducta adaptativa en deficientes mentales. (Tesis de licenciatura, Universidad Michoacana de San Nicolás de Hidalgo, México). Recuperado de http:// bibliotecavirtual.dgb.umich.mx:8083/jspui/ bitstream/123456789/6640/1/FUNCIONAMIENTOFAMILIARYCONDUCTAADAPTATIVAENDEFICIENTESMETALES.pdf

Instituto Nacional de Estadística (INE). (2004). Primer Estudio Nacional de la Discapacidad e Informes Regionales. Fondo Nacional de la Discapacidad. Recuperado de http://www. ine.cl/canales/chile_estadistico/encuestas_ discapacidad/discapacidad.php

Krause, A. y Román, M. (2012). Proyecto de investigación D112-0077: propiedades psicométricas de la versión española del Inventario para la Planificación de Servicios y la Programación Individual (ICAP), en escolares de la ciudad de Temuco. Universidad de La Frontera.

Lotito, F. y Sanhueza, H. (2011). Discapacidad y barreras arquitectónicas: un desafío para la inclusión. Revista AUS, (9), 10-13.

Luckasson, R., Borthwick-duffy, S., Buntix, W., Coulter,D., Craig, E., Reeve, A. \& cols. (2002). Mental retardation. Definition, classification and systems of supports. Washington, D. C. United States: American Association in Mental Retardation.
Ministerio de Educación. (2009). Orientaciones técnicas para la evaluación diagnóstica de estudiantes que presentan NEE asociadas a discapacidad intelectual. Santiago, Chile: Unidad de Educación Especial.

Medina, M. (2010). Evaluación de la conducta adaptativa de las personas con discapacidad intelectual. Valoración y usos de las escala $A B S-R C:$ 2. (Tesis de doctorado). España: Universidad de Burgos.

Montero, D. (1996). Evaluación de la conducta adaptativa en personas con discapacidad. Adaptación y validación del ICAP. Bilbao: ICE. Universidad de Deusto-Mensajero.

Navas, P., Verdugo, M., Arias, B. y Gómez, L. (2010). La conducta adaptativa en personas con discapacidad intelectual. Siglo Cero, 41(235), 28-48.

Salkind, N. (1998). Métodos de Investigación. México: Prentice Hall.

Schalock, R., Luckasson, R., Shogren, K., Borthwick-duffy, S., Bradley, V., Buntinx, W., Coulter, D., Craig, T., Gomez, S., Lachapelle, Y., Reeve, A., Snell, M., Spreat, S.,Tasse, M., Thompson, J., Verdugo, M., Wehmeyer, M. y Yeager, M. (2007). El Nuevo concepto de retraso mental: Comprendiendo el cambio del término discapacidad intelectual. Siglo Cero, $38(4), 5-20$.

Visauta, B. (2007). Análisis estadístico con SPSS 14. Madrid: McGraw-Hill. 